\title{
Redshift-Distance Survey of Early-Type Galaxies: Dipole of the Velocity Field
}

\author{
L. N. da Costa ${ }^{1,2}$, M. Bernardi ${ }^{1,3,4}$, M. V. Alonso ${ }^{5}$, G. Wegner ${ }^{6}$ \\ C. N. A. Willmer ${ }^{2,7}$, P. S. Pellegrini ${ }^{2}$, M. A. G. Maia ${ }^{2}$, S. Zaroubi ${ }^{4}$
}

\begin{abstract}
We use the recently completed redshift-distance survey of nearby early-type galaxies (ENEAR) to measure the dipole component of the peculiar velocity field to a depth of $c z \sim 6000 \mathrm{kms}^{-1}$. The sample consists of 1145 galaxies brighter than $m_{B}=14.5$ and $c z \leq 7000 \mathrm{kms}^{-1}$, uniformly distributed over the whole sky, and 129 fainter cluster galaxies within the same volume. Most of the $D_{n}-\sigma$ distances were obtained from new spectroscopic and photometric observations conducted by this project, ensuring the homogeneity of the data over the whole sky. These 1274 galaxies are objectively assigned to 696 objects - 282 groups/clusters and 414 isolated galaxies. We find that within a volume of radius $\sim 6000 \mathrm{kms}^{-1}$, the best-fitting bulk flow has an amplitude of $\left|\mathbf{v}_{\mathbf{b}}\right|=220 \pm 42 \mathrm{kms}^{-1}$ in the CMB restframe, pointing towards $l=304^{\circ} \pm 16^{\circ}$, $b=25^{\circ} \pm 11^{\circ}$. The error in the amplitude includes statistical, sampling and possible systematic errors. This solution is in excellent agreement with that obtained by the SFI
\end{abstract}

\footnotetext{
${ }^{1}$ European Southern Observatory, Karl-Schwarzschild Strasse 2, D-85748 Garching, Germany

${ }^{2}$ Observatório Nacional, Rua General José Cristino 77, Rio de Janeiro, R. J., 20921, Brazil

${ }^{3}$ Universitäts-Sternwarte München, Scheinerstr. 1, D-81679, München, Germany

${ }^{4}$ Max Planck Institut für Astrophysik, Karl-Schwarzschild Strasse 1, D-85740, Garching, Germany

${ }^{5}$ Observatorio Astrónomico de Córdoba, Laprida 854, Córdoba, 5000, Argentina

${ }^{6}$ Department of Physics \& Astronomy, Dartmouth College, Hanover, NH 03755-3528, USA

${ }^{7}$ UCO/Lick Observatory, University of California, 1156 High Street, Santa Cruz, CA 95064, USA
} 
Tully-Fisher survey. Our results suggest that most of the motion of the Local Group is due to fluctuations within $6000 \mathrm{kms}^{-1}$, in contrast to recent claims of large amplitude bulk motions on larger scales.

Subject headings: Cosmology: large-scale structure of universe - cosmology: observations - galaxies: distances and redshifts

\section{Introduction}

Within the gravitational instability framework for the growth of cosmic structures, the peculiar velocity field of galaxies and clusters is a direct probe of density fluctuations of the underlying mass distribution. Among several possible statistics that can be used, measurements of the bulk motion amplitude on different scales are the simplest and provide, at least in principle, constraints on the power-spectrum of mass fluctuations. This has motivated several attempts to measure the dipole component of the local peculiar velocity field and to determine the volume within which the streaming motion vanishes in the restframe defined by the Cosmic Microwave Background radiation (CMB). At this distance the distribution of matter within the encompassing volume should explain the $\sim 600 \mathrm{kms}^{-1}$ motion of the Local Group relative to the CMB restframe.

Observational searches of large-scale flows date back to the pioneering work of Rubin et al. (1976). Since then, redshift-distance surveys have greatly expanded, the data quality has improved significantly, and several recent attempts have been made using different techniques and samples (e.g., Strauss \& Willick 1995). Despite these efforts, the results remain to a large extent controversial. The original claim that the flowfield out to $c z \sim 4000 \mathrm{kms}^{-1}$ is characterized by a coherent, large-amplitude $\sim 500 \mathrm{kms}^{-1}$ streaming motion (Dressler et al. 1987) relative to the CMB was revised to incorporate a large concentration of mass, the so-called Great Attractor (hereafter GA), near $l=310^{\circ}, b=10^{\circ}$ (Lynden-Bell et al. 1988). More recent claims for the existence of a large amplitude flow $\sim 600 \mathrm{kms}^{-1}$, with a coherence length of $\sim 100 h^{-1} \mathrm{Mpc}$ (e.g., Willick 1990; Mathewson, Ford \& Buchhorn 1992), suggesting excess power on very large scales, have also received reconsideration from the following standpoints. First, a careful re-analysis of the available data yielded a significantly smaller bulk velocity (Courteau et al.1993). Second, the analysis of the independent SFI ( $I$-band field spiral) TF-survey led to a different characterization of the flowfield. Indeed, the SFI velocity field shows that the flow is not as coherent as originally envisioned, exhibiting along the Supergalactic Plane a bifurcation towards the Great Attractor and Perseus-Pisces, similar to that predicted from reconstructions of the $I R A S$ surveys (e.g., da Costa et al. 1996). Furthermore, the flow within $6000 \mathrm{kms}^{-1}$ is characterized by a strong shear across the volume, in contrast to the picture of a coherent motion of all structures. Recent analyses based on the re-calibrated Mark III catalogs lead to a roughly consistent picture to that obtained with SFI (da Costa et al. 1996; Dekel et al. 1999), even though some discrepancies still remain. For instance, Mark III yields a systematically larger amplitude of the bulk motion $\sim 370 \pm 110 \mathrm{kms}^{-1}$ on scales 
$\sim 5000 \mathrm{kms}^{-1}$ as compared to values $\lesssim 300 \mathrm{kms}^{-1}$ obtained by applying different techniques to the SFI sample (da Costa et al.1996; Giovanelli et al.1998a). In particular, a direct fit to the SFI radial velocities yields a bulk velocity of $200 \pm 65 \mathrm{kms}^{-1}$ within the sphere of radius $\sim 6500 \mathrm{kms}^{-1}$ consistent with that obtained from the SCI cluster sample (Giovanelli et al. 1998b). These results suggest that a significant fraction of the LG motion is generated on scales $\lesssim 6000 \mathrm{kms}^{-1}$. While recent direct measurements of the bulk velocity on larger scales (Dale et al. 1999) suggest that this may indeed be the case, other works (Lauer \& Postman 1994; Willick 1999; Hudson et al. 1999)

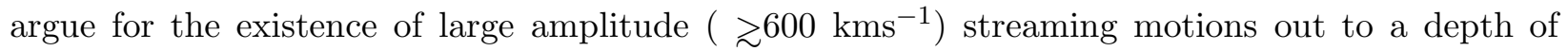
$15,000 \mathrm{kms}^{-1}$.

In this paper we use the recently completed all-sky, homogeneous redshift-distance survey of early-type galaxies (ENEAR, da Costa et al. 2000, hereafter Paper I) to study the dipole component of the peculiar velocity field within $c z \lesssim 6000 \mathrm{kms}^{-1}$. Our main goal is to compare our results using an entirely independent sample to those obtained by existing Tully-Fisher surveys.

\section{The Sample}

In the present analysis, we use the ENEAR redshift-distance survey described in greater detail in Paper I of this series. Briefly, the ENEAR sample consists of 1600 early-type galaxies brighter than $m_{B}=14.5$ and with $c z \leq 7000 \mathrm{kms}^{-1}$, with $D_{n}-\sigma$ distances available for 1359 galaxies. Of these 1145 were deemed suitable for peculiar velocity analysis (Paper I). To the magnitudelimited sample we added 285 galaxies fainter and/or with redshifts $>7000 \mathrm{kms}^{-1}$, 129 within the same volume as the magnitude-limited sample. In total, the cluster sample consists of 569 galaxies in 28 clusters, which are used to derive the distance relation. Over $80 \%$ of the galaxies in the magnitude-limited sample and roughly $60 \%$ of the cluster galaxies have new spectroscopic and R-band photometric data obtained as part of this program. Furthermore, repeated observations of several galaxies in the sample provide overlaps between observations conducted with different telescope/instrument configurations and with data available from other authors. These overlaps are used to tie all measurements into a common system, thereby ensuring the homogeneity of the entire dataset. In contrast to other samples new observations conducted by the same group are available over the entire sky. The comparison between the sample of galaxies with distances and the parent catalog also shows that the sampling across the sky is uniform.

Individual galaxy distances were estimated from a direct $D_{n}-\sigma$ template relation derived by combining all the available cluster data, corrected for incompleteness and associated diameterbias (Lynden-Bell et al. 1988). The construction of the template relation was carried out following Giovanelli et al. (1997). From the observed scatter of the template relation the estimated fractional error in the inferred distance of a galaxy is $\Delta \sim 0.19$, nearly independent of the velocity dispersion.

Since early-type galaxies are found preferentially in high-density regions, galaxies have been assigned to groups/clusters using well-defined criteria imposed on their projected separation and 
velocity difference relative to the center of groups and clusters, as described in paper I. Early-type galaxies in a group/cluster are replaced by a single object having: (1) the redshift given by the group's mean redshift, which is determined considering all morphologies; (2) the distance given by the error-weighted mean of the inferred distances, for groups with two or more early-types; and (3) the fractional distance error given by $\Delta / \sqrt{(} N)$, where $N$ is the number of early-types in the group. In some cases groups were identified with Abell/ACO clusters within the same volume as the ENEAR sample and fainter cluster galaxies were added, as described in Paper I.

The inferred distances are corrected for the homogeneous and inhomogeneous Malmquist bias (IMB). The latter was estimated using the PCSz density field (Branchini et al. 1999), corrected for peculiar velocity effects, following Willick et al. (1997). In this calculation we also include the correction for the sample redshift limit. It should be noted that this is an approximation as earlytypes are biased relative to $I R A S$ galaxies. A complete description of the sample used and the corrections applied will be presented in a subsequent paper of this series. As an illustration of the velocity field mapped by the ENEAR objects we show in Figure 1 the projected distribution of objects in Galactic coordinates with the sample split into different distance shells. The different symbols distinguish between objects with positive (crosses) and negative peculiar velocities (circles). The peculiar velocities are relative to the CMB restframe and have been computed from fully corrected distances as described above. For an alternative view of the data we refer the reader to paper I. In Figure 1 structures such as the GA at $l \sim 300^{\circ}, b \sim 30^{\circ}$ and the Perseus-Pisces (PP) complex at $l \sim 120^{\circ}, b \sim-40^{\circ}$ are easily recognized in the two outermost shells. Note that in these directions one finds evidence of outflow and infall as expected around mass concentrations. As will be shown in a later paper of this series, the presence of mass concentration in the PP region is confirmed from the reconstruction of the three-dimensional velocity field and mass distribution which shows that both structures have comparable peak density contrasts. The prominence of the PP complex is perhaps the most significant difference between the reconstructions based on the ENEAR and the 7S samples. The ENEAR reconstructed fields are also in good agreement with those obtained from the PSCz redshift survey (Branchini et al. 1999), corrected for peculiar velocities, as it will be shown in a forthcoming paper.

\section{Measurements of the Bulk Motion}

One of the primary goals of the ENEAR survey has been to investigate the robustness of previous peculiar velocity analyses using an independent and uniform sample of early-type galaxies probing a comparable volume as the recently completed TF-surveys. While many tests are possible and will be explored in more detail in separate papers (e.g., Borgani et al. 2000), here we consider the dipole component of the velocity field. A bulk flow model is the simplest way to globally characterize the velocity field, having been extensively used in previous work (e.g., Dekel 1999 for a recent review). To determine the best-fitting bulk flow we minimize (e.g., Lynden-Bell et al. 1988)

$$
\chi^{2}=\sum w_{i}\left(u_{i}-\mathbf{v}_{b} \cdot \hat{\mathbf{r}}_{i}\right)^{2}
$$


where $u_{i}$ is the radial component of the peculiar velocity of the $i^{t h}$ object in the CMB restframe, located in the direction $\hat{\mathbf{r}}_{i}, \mathbf{v}_{b}$ is the bulk flow and $w_{i}$ is the weight given to the $i^{\text {th }}$ object in the sample. In our calculations we use either uniform (equal) weights $w_{i}=1$ or $w_{i}=\frac{1}{\epsilon_{i}^{2}+\sigma^{2}}$, where $\epsilon_{i}$, is the sum in quadrature of the distance and redshift errors (the latter is negligible in the case of field objects), and $\sigma$ is the one-dimensional velocity dispersion due to true velocity noise generated on small scales.

Table 1 summarizes the bulk flow results obtained using various sub-samples extracted from the combined sample of 696 objects within different volumes. The table gives for each volume of radius $R$ in units of $\mathrm{kms}^{-1}$, the number of objects in each sub-sample, the amplitude and direction of the best-fitting bulk motion, and their respective errors, obtained using different weighting schemes. The amplitude of the bulk motion is relative to the CMB restframe and its direction is expressed in terms of the galactic longitude and latitude. The errors were estimated from 1000 Monte-Carlo realizations generated by adding Gaussian random deviates of the distance errors to the original distances, from which the dispersion of the dipole components are calculated. In the table, the weighted solutions assume a thermal component of $\sigma_{f}=250 \mathrm{kms}^{-1}$ which is combined with the object's distance errors in quadrature. The bulk amplitudes listed in Table 1 have been corrected for the error-bias obtained subtracting from the square of the best-fitting value of the bulk velocity the sum in quadrature of the errors in each Cartesian component (Lauer \& Postman 1994). The amplitude of this correction is relatively small $\sim 50 \mathrm{kms}^{-1}$. We point out that the amplitude of the bulk velocity at $6000 \mathrm{kms}^{-1}$ is insensitive to the Malmquist bias correction. The comparison between the results obtained using raw distances, with those corrected only for homogeneous Malmquist bias (HMB) and those obtained using the full correction, are comparable to the estimated errors in the bulk velocity. Typical values for the HMB and IMB corrections are $13 \%$ and $4 \%$, respectively. Only the direction of the dipole shows some dependence on the adopted correction. In particular, neglecting the IMB correction yields lower values of $b$. The good agreement between the direction of the fully corrected ENEAR and those of SFI and Mark III, using different procedures to estimate the IMB, is reassuring.

From the direct fit of the radial velocities using equal weights we find $\left|\mathbf{v}_{b}\right|=220 \pm 42$ in the direction $l=304^{\circ} \pm 16^{\circ}, b=25^{\circ} \pm 11^{\circ}$ within a radius of $c z \sim 6000 \mathrm{kms}^{-1}$. Note that this value is smaller than the preliminary value reported earlier by Wegner et al. (1999) which was not corrected for the error-bias and was determined before the full sample had been assembled. A somewhat larger value is obtained when objects are weighted by their distance errors, but the amplitude is still less than $300 \mathrm{kms}^{-1}$ and essentially in the same direction. The direction of the ENEAR dipole is compared in Figure 2 to other recent estimates measured on similar scales $\left(\sim 5000-6500 \mathrm{kms}^{-1}\right)$ using the SFI (Giovanelli et al. 1998a) and the revised Mark III (Dekel et al. 1999) samples. The contours represent the 1-3 $\sigma$ confidence levels, derived from the Monte-Carlo simulations. Perhaps the most interesting result is the excellent agreement both in direction and amplitude between the ENEAR and SFI dipole solutions, probably the two most homogeneous allsky samples currently available for the analysis of peculiar velocity data. Particularly important is 
the well known fact that early-type (E and S0) and late-type (Sc) galaxies probe distinct regions of the galaxy distribution - while spirals are found predominantly in low-density regions and are more uniformly distributed, the distribution of ellipticals is clumpier, delineating more clearly the most prominent nearby structures. Equally important is the fact that the peculiar velocities used in the two studies are based on independent distance relations involving different measurements and corrections. In Figure 2 we also show the direction of the dipoles recently measured on larger scales. The results obtained on scales of $\sim 6000 \mathrm{kms}^{-1}$ are consistent, both in direction and amplitude, with those measured on much larger scales using the SCI+SCII sample. Combined these results suggest that while most of the LG motion stems from fluctuations within $6000 \mathrm{kms}^{-1}$ some contribution also comes from larger scales where a better agreement between the dipole direction and the LG motion is found. It is important to note, however, that currently there is very little agreement among various determinations of the dipole on scales $\gtrsim 10,000 \mathrm{kms}^{-1}$.

To evaluate the possible impact of sampling effects directly from the data, we have also computed the dipole solution splitting the sample into field galaxies and groups/clusters. We find that for $R \sim 6000 \mathrm{kms}^{-1}$ these sub-samples yield bulk velocities of $\sim 175 \mathrm{kms}^{-1}$ for groups/clusters and $\sim 240 \mathrm{kms}^{-1}$ for field galaxies, with errors of the order of $\sim 70 \mathrm{kms}^{-1}$. These velocities are somewhat higher $\left(\sim 300 \mathrm{kms}^{-1}\right)$ when the objects are weighted by their distance error. However, in this case the mean weighted depth is small, for instance, $\sim 2400 \mathrm{kms}^{-1}$ in the case of field galaxies. The results obtained for field galaxies and groups/clusters are, individually, in good agreement with the amplitude and direction of the dipole obtained from TF surveys (Giovanelli et al. 1998a). We conclude that on scales of $\sim 6000 \mathrm{kms}^{-1}$ the sampling error is small and comparable to the estimated random error of the bulk velocity $\left(\lesssim 40 \mathrm{kms}^{-1}\right)$. Adding this value in quadrature to that estimated from the simulations we estimate the random error to be $\sim 60 \mathrm{kms}^{-1}$. Another potential source of error in the bulk velocity are systematic uncertainties in the distance arising from mismatches in the velocity dispersion scale. Typically, the correction applied to $\sigma$ for different runs is less than 0.020 dex with an uncertainty of 0.009-0.018 dex, which in principle could lead to large errors in the amplitude of the bulk flow. However, given the large number of runs covering each region of sky and the fact that the observed galaxies in each run were selected randomly, we estimate this contribution to be at most $\sim 45 \mathrm{kms}^{-1}$ in each hemisphere. On the other hand, the uncertainty in the offset between measurements of the velocity dispersion from northern and southern observations is estimated to be $\lesssim 0.006 \mathrm{dex}$, as determined from the a sample of galaxies observed from both hemispheres. This uncertainty corresponds to about $1.5 \%$ in distance or to $50 \mathrm{kms}^{-1}$, which we take as an an upper limit to the systematic error in the measured bulk velocity.

\section{Conclusions}

Using a sample of 1274 early-type galaxies in 696 objects comprising 414 isolated galaxies and 282 groups/clusters, drawn from the recently completed all-sky ENEAR redshift-distance survey, we have computed the dipole component of the local velocity field to a depth of $\sim 6000 \mathrm{kms}^{-1}$. 
Our main conclusion is that the streaming motion amplitude of the ensemble of galaxies within the largest volume considered is small $\sim 200 \mathrm{kms}^{-1}$. Similar small amplitudes are obtained when the sample is split into isolated galaxies and groups/clusters, indicating that sampling effects are relatively minor on these scales. The amplitude and direction of the ENEAR dipole agree well with the results obtained from similar analysis using the SFI TF-survey. This is a remarkable result since these samples consider galaxies of different morphological types sampling different regions of space, were selected using different criteria, and the peculiar velocities are derived using different distance relations. Small bulk velocities have also recently been obtained using new TF data (Courteau et al. 2000) as well as other distance indicators (see Dekel 1999 for a review). If these results are confirmed, the peculiar velocity field observed locally can easily be accounted for by the currently popular cosmological models.

The authors would like to thank C. Rité and O. Chaves for their contribution over the years. Special thanks to E. Branchini. The bulk of the data used in this paper were obtained from observations conducted at the European Southern Observatory (ESO) and the MDM Observatory.

\section{REFERENCES}

Borgani, S., Bernardi, M., da Costa, L. N., Wegner, G., Alonso, M. V., Willmer, C. N. A., Pellegrini, P. S. \& Maia, M. A. G., 2000, ApJ, in press

Branchini, E., Teodoro, L., Frenk, C. S., et al. 1999, MNRAS, 308, 1

Courteau, S., Faber, S. M., Dressler, A., \& Willick, J. A. 1993, ApJ, 412, L51

Courteau, S., Willick, J. A., Strauss, M. A., Schlegel, D., \& Postman, M, 2000, ApJ, submitted (astro-ph/0002420)

da Costa, L. N., Freudling, W., Wegner, G., Giovanelli, R., Haynes, M. P., \& Salzer, J. J. 1996, ApJ, 468, L5

da Costa, L. N., Bernardi, M., Alonso, M. V., Wegner, G., Willmer, C. N. A., Pellegrini, P. S., Rité, C., \& Maia, M. A. G. 2000, AJ, in press (Paper I)

Dale, D. A., Giovanelli, R., Haynes, M. P., Campusano, L. E., Hardy, E., \& Borgani, S. 1999, ApJL, 510, 11

Dekel, A., Eldar, A., Kolatt, T., Yahil, A., Willick, J. A., Faber, S. M., Courteau, S., \& Burstein, D. 1999, ApJ, 522, 1

Dekel, A., in Cosmic Flows: Towards and Understanding of Large-Scale Structure, eds. S. Courteau, M. A. Strauss, \& J. A. Willick, ASP Conf. Ser., astro-ph/9911501

Dressler, A., Lynden-Bell, D., Burstein, D., Davies, R. L., Faber, S. M., Terlevich, R., \& Wegner, G. 1987, ApJ, 313, 42 
Giovanelli, R., Haynes, M. P., Herter, T., Vogt, N. P. da Costa, Freudling, W.,L. N., Salzer, J. J., \& Wegner, G, 1997, AJ, 113,53

Giovanelli, R., Haynes, M. P., Freudling, W., da Costa, L. N., Salzer, J. J., \& Wegner, G. 1998a, ApJ, 505, L91

Giovanelli, R., Haynes, M. P., Salzer, J. J., Wegner, G., da Costa, L. N., \& Freudling, W. 1998b, AJ, 116, 2632

Hudson, M. J., Smith, R. J., Lucey, J. R., Schlegel, D. J., \& Davies, R. L. 1999, ApJ, 512, L79

Lauer, T. R., \& Postman, M. 1994, ApJ, 425, 418

Lynden-Bell, D., Faber, S. M., Burstein, D., Davies, R. L., Dressler, A., Terlevich, R., \& Wegner, G. 1988, ApJ, 326, 19

Mathewson, D. S., Ford, V. L., \& Buchhorn, M. 1992, ApJS, 81, 413

Riess, A. G., Davis, M., Baker, J., Kirshner, R. P. 1997, ApJ, 488, 1

Rubin, V. C., Roberts, M. S., Thonnard, N., Ford, W. K., 1976, AJ 81, 719

Strauss, M. A., \& Willick, J. A. 1995, Physics Reports, 261, 271

Wegner, G. et al. 1999, in Cosmic Flows: Towards and Understanding of Large-Scale Structure, eds. S. Courteau, M. A. Strauss, \& J. A. Willick, ASP Conf. Ser., astro-ph/9908354

Willick, J. A. 1990, ApJ, 351, L5

Willick, J. A., Courteau, S., Faber, S. M., Burstein, D., Dekel, A., \& Strauss, M. A. 1997, ApJS, 109, 333

Willick, J. A. 1999, ApJ, 522, 647 

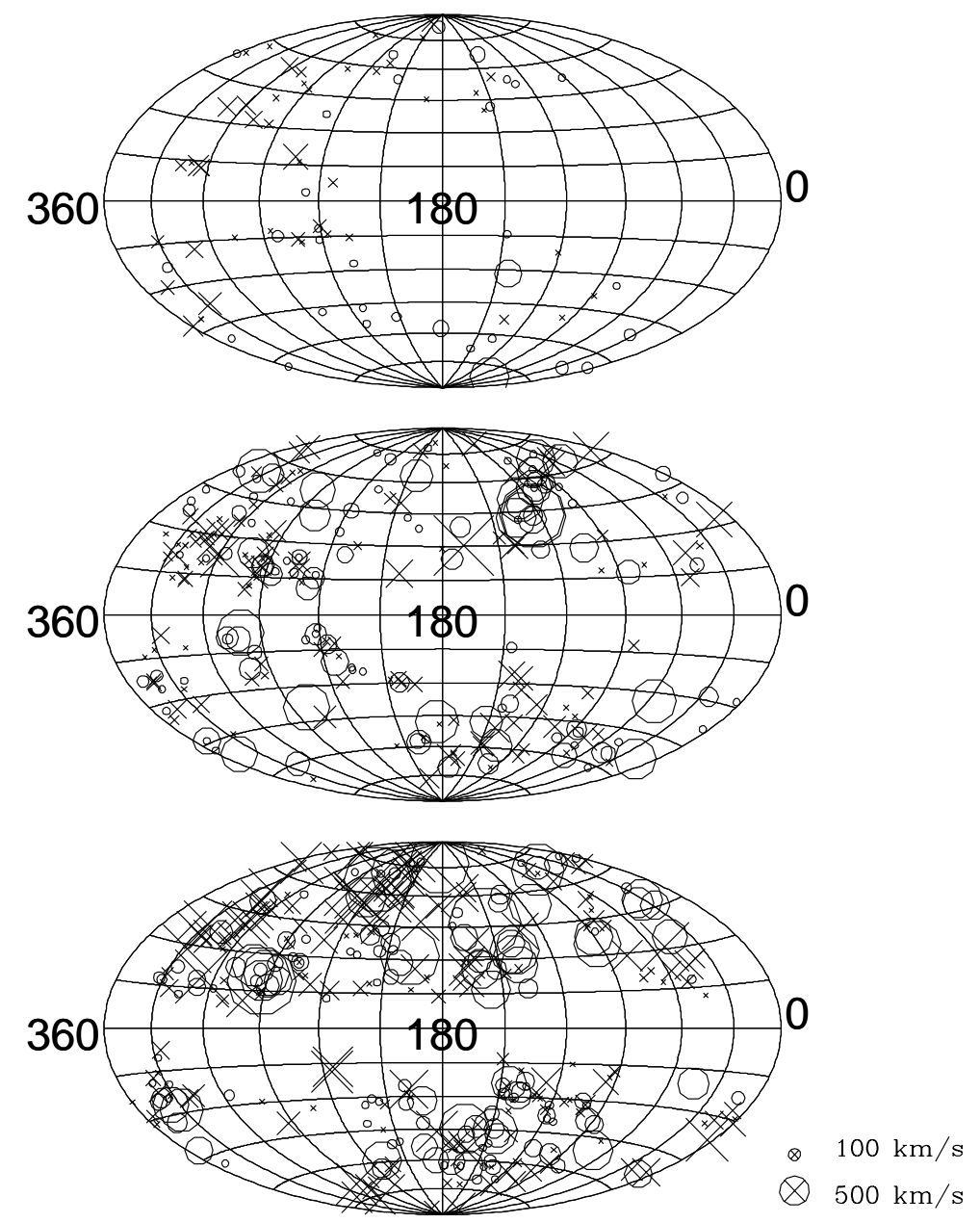

Fig. 1.- Projected distribution in Galactic coordinates of the ENEAR peculiar velocity field in different distance shells $2000 \mathrm{kms}^{-1}$ thick in the interval $0<R<6000 \mathrm{kms}^{-1}$. The velocities are relative to the CMB resframe and the different symbols represent infall (open circles) and outflow (crosses). Their sizes are proportional to the galaxy's peculiar velocity amplitude. 


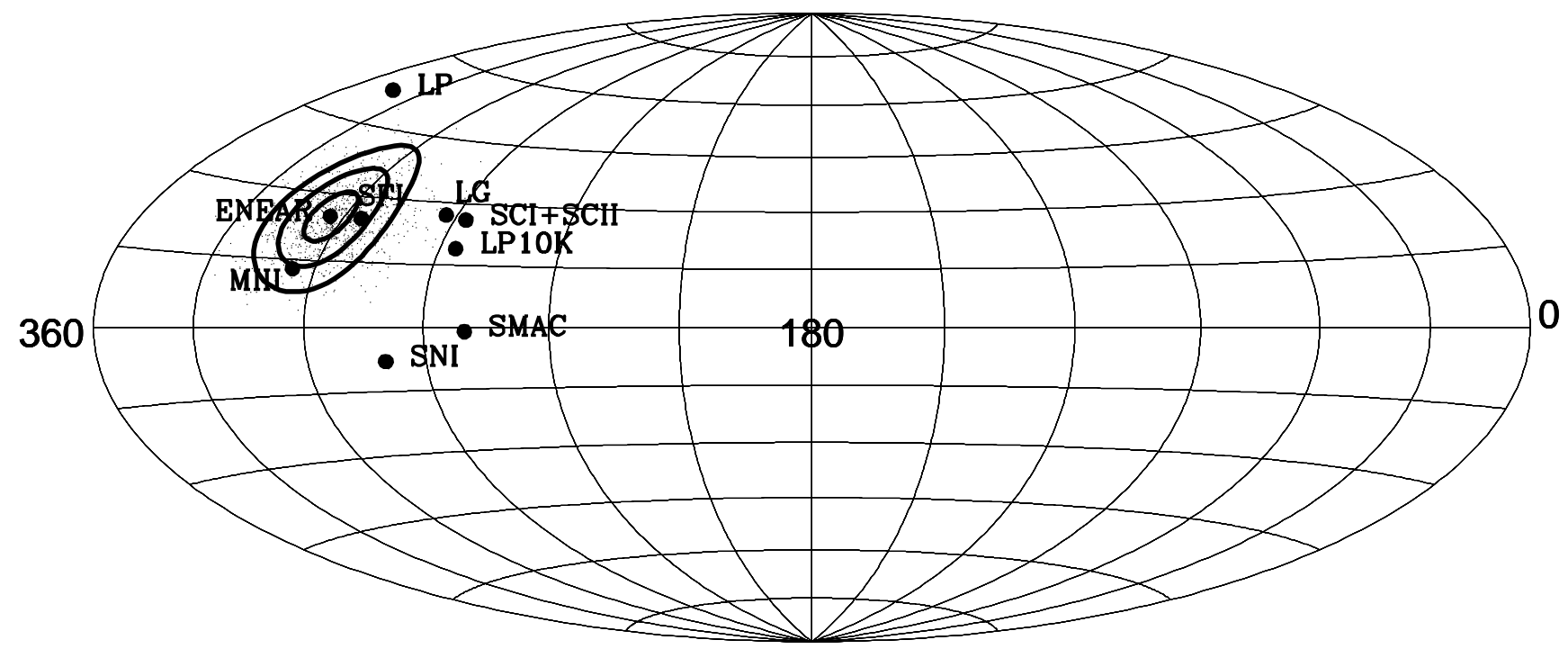

Fig. 2. - The bulk flow direction in Galactic coordinates and the direction obtained from 1000 Monte-Carlo realizations (dots). The contours represent 1, 2, and $3 \sigma$ error ellipsoids as derived from the Monte-Carlo realizations. The figure shows the direction of the LG motion (LG) and the dipole directions obtained by other authors on different scales (see text). We adopt the following notation: LP (Lauer \& Postman 1994); MIII (Dekel et al. 1999); SFI (Giovanelli et al. 1998a); LP10K (Willick 1999); SCI+SCII (Dale et al. 1999); SNI (Riess et al.1997); SMAC (Hudson et al. 1999). 
Table 1: Dipole Component of the Velocity Field

\begin{tabular}{|c|c|c|c|c|c|c|c|}
\hline Sample & $N$ & $\begin{array}{c}\left|\mathbf{v}_{b}\right| \\
\left(\mathrm{kms}^{-1}\right)\end{array}$ & $\begin{array}{c}1 \\
\text { (degree) }\end{array}$ & $\begin{array}{c}\mathrm{b} \\
(\text { degree })\end{array}$ & $\begin{array}{c}\left|\mathbf{v}_{b}\right| \\
\left(\mathrm{kms}^{-1}\right)\end{array}$ & $\begin{array}{c}1 \\
\text { (degree) }\end{array}$ & $\begin{array}{c}\mathrm{b} \\
\text { (degree) }\end{array}$ \\
\hline Objects & & & UNIFORM & & & WEIGHTED & \\
\hline$R<2000 \mathrm{kms}^{-1}$ & 77 & $442 \pm 97$ & $310 \pm 16$ & $21 \pm 10$ & $446 \pm 78$ & $308 \pm 14$ & $23 \pm 8$ \\
\hline$R<4000 \mathrm{kms}^{-1}$ & 324 & $147 \pm 62$ & $306 \pm 18$ & $9 \pm 14$ & $350 \pm 47$ & $301 \pm 10$ & $16 \pm 7$ \\
\hline$R<6000 \mathrm{kms}^{-1}$ & 656 & $220 \pm 42$ & $304 \pm 16$ & $25 \pm 11$ & $298 \pm 38$ & $299 \pm 10$ & $18 \pm 7$ \\
\hline
\end{tabular}

